\title{
Comparações sonoras e morfométricas entre populações de Arremon taciturnus no Nordeste do Brasil
}

\author{
Wilmara Guedes Lucenaa, Erich de Freitas Mariano ${ }^{a}$ \\ a Unidade Acadêmica de Ciências Biológicas, Universidade Federal de Campina Grande, Patos, 58708-110, Paraíba, Brasil. *lucenawg@gmail.com
}

Recebido: 27 junho 2017 / Aceito: 02 novembro 2017 / Publicado online: 20 janeiro 2018

\begin{abstract}
Resumo
Estudos recentes apontam que as florestas estacionais do nordeste brasileiro são formadas por duas unidades biogeográficas distintas, uma relacionada a Floresta Atlântica costeira e outra com os domínios da caatinga. Esta pesquisa pretendeu verificar se existe algum grau de diferenciação morfométrica e acústica entre as populações de Arremon taciturnus (Tico-tico-de-bicopreto) em florestas de altitude no Nordeste do Brasil, localizadas a leste e a oeste do planalto da Borborema. Com esta finalidade foram realizados testes paramétrico (teste t) e não paramétrico (teste U), de acordo com a normalidade dos dados acústicos, analisando se existe diferença significativa entre os parâmetros vocais das populações. Para verificar se existem diferenças biométricas entre as áreas estudadas foi realizada uma análise discriminante e de correspondência. Estas, por sua vez, não apresentaram diferença. Entretanto, as análises bioacústicas apontaram diferenças em parâmetros do canto como número de notas e em parâmetros como duração da $1^{\mathrm{a}}, 2^{\mathrm{a}}$, e $3^{\mathrm{a}}$ nota da segunda parte do canto. A. taciturnus é uma espécie que possui capacidade para aprendizado do canto. Desta forma, as diferenças encontradas em alguns parâmetros vocais pode ser indício de uma variação interespecífica nas populações, chamadas de dialetos vocais.
\end{abstract}

Palavras-chave: Bioacústica, Florestas estacionais Semideciduais Montanas, Taxonomia.

\section{Song and morphometric comparison among Arremon taciturnus populations in Northeastern Brazil}

\begin{abstract}
Recent studies indicate that the seasonal forests of the Brazilian Northeast are formed by two distinct biogeographic units, one related to the Atlantic Coastal Forest and another to the caatinga domains. This aim intended to verify if there is any degree of morphometric and acoustic differentiation between the populations of Arremon taciturnus (Tico-tico-de-bico-preto) in high altitude forests in the Northeast of Brazil, located to the east and west of the Borborema plateau. For this purpose, parametric (t-test) and non-parametric (U-test) tests were performed according to the normality of the acoustic data, analyzing whether there is a significant difference between the vocal parameters of the populations. To verify if there are biometric differences between the studied areas a discriminant and correspondence analysis was performed. These, in turn, showed no difference. However, bioacoustic analysis pointed to differences in singing parameters such as number of notes and parameters such as duration of $1^{\mathrm{a}} 2^{\mathrm{a}}$ and $3^{\mathrm{a}}$ notes of the second part of the song. A. taciturnus is a species that has the ability to learn the song. In this way, the differences found in some vocal parameters may be indicative of an interspecific variation in the populations, called vocal dialects.
\end{abstract}

Keywords: Bioacoustics, Seasonal Forests Semideciduous Mountains, Taxonomy.

\section{Introdução}

A biacústica tem se tornado uma ciência muito importante e adequada para detectar e confirmar as diferenças entre as espécies de aves e fornece técnicas rápidas e de baixo custo (Tubaro, 1999). De acordo com Vielliard (1987), a principal função biológica do canto é o reconhecimento específico, podendo assim ser definido como canto o sinal de comunicação sonora que contém esta informação (reconhecimento). Além disso, o canto segue uma evolução filogenética e por esse motivo pode ser utilizado para verificar as relações evolutivas entre as diferentes populações de espécies de aves (Vielliard, 1997; Silva, 2011). Em consequência disso, o estudo do canto dos pássaros tem sido um assunto muito importante para análises de variação geográfica e evolução cultural de populações de aves (Shieh e Liang, 2007).

Devido a sua extrema versatilidade podemos encontrar diferenças no canto das aves ao longo do espaço geográfico (Silva, 2011). No entanto, em aves que não aprendem suas músicas, as mesmas canções ou vocalizações tendem a ocorrer 
em grandes extensões geográficas, presumivelmente porque os genes que definem os cantos ocorrem sobre aquelas mesmas extensões (Marler, 2004). Já em aves que aprendem seus cantos pela imitação de seus tutores, um maior grau de variação geográfica é esperado, pelo fato de haver rápida evolução cultural entre populações (Kroodsma e Baylis, 1982; Mundiger, 1982), como acontece com espécies da subordem Passeri ("oscines") (ordem passeriformes) (Saranathan et al., 2007). Segundo Marler (2004), uma vocalização diferenciada pode ter um papel fundamental no processo de especiação quando diferenças acústicas entre as populações afetam a dispersão e cruzamento.

O tico-tico-de-bico-preto, A. taciturnus (Hermann, 1783), possui três subespécies atualmente reconhecidas: $A$. taciturnus taciturnus, A. taciturnus nigrirostris e A. taciturnus axillaris (Buainain, 2017). A subespécie A. taciturnus taciturnus é uma ave que vive nas porções mais próximas ao solo de florestas húmidas e bosques, ocorrendo do leste da Colômbia até o sul da Venezuela, nas Guianas e no Brasil, podendo ser encontrado na região central da Amazônia brasileira, nos estados de Tocantins, Goiás e no nordeste, atingindo até os estados de Minas Gerais e Espírito Santo. É encontrado nas faixas altitudinais próximas aos $1000 \mathrm{~m}$ até os $1500 \mathrm{~m}$ (Ridgely e Tudor, 2009). É uma espécie que possui um tipo de canto simples com dois tipos de frases: uma introdutória com notas curtas e agudas e uma frase principal com notas mais longas como assobios, variando na modulação, mas em sua maioria é um pouco descendente (Buainain, 2017).

A hipótese proposta por Rodal et al. (2008), na qual é apontado que as florestas de altitude no nordeste do Brasil seriam compostas por dois grupos florísticos biogeograficamente diferentes contrapõe a hipótese inicial de Andrade-Lima (1982), de que os chamados brejos de altitude seriam remanescentes de mata atlântica encravados nos domínios da Caatinga. Para essa hipótese, Rodal et al. Propõe que o Planalto da Borborema seja a barreira geográfica para essas formações florestais. Para as aves, Mariano (2014) encontraram o mesmo padrão, grupando as formações florestais a oeste da Borborema com as formações de mata seca e as formações à leste do planalto com a floresta litorânea.

Desta forma, populações de A. taciturnus podem apresentar divergências genéticas e fenotípicas, o que justifica uma investigação taxonômica para as espécies destes enclaves florestais. O presente trabalho objetivou verificar se a hipótese da Borborema como uma barreira biogeográfica para estas formações, sugerida por Rodal et al. (2008) e suportada por Mariano (2014), proporcionou modificações acústicas e biométricas entre as populações de A. taciturnus.

\section{Materiais e métodos}

\section{Espécie modelo}

Atualmente, o gênero Arremon conta com 11 espécies conhecidas na América latina. Dentre estas, 4 espécies ocorrem no Brasil: A. taciturnus, de hábito florestal e que ocupa o sub-bosque de áreas na Amazônia, Floresta Atlântica do nordeste e as áreas de florestas estacionais interioranas;
Arremon flavirostris (Swainson, 1838) Tico-tico-de-bicoamarelo, distribuído pelo Brasil central até o sudeste do Paraguai e nordeste da Argentina; Arremon semitorquatus (Swainson, 1838) Tico-tico-do-mato, que se distribui na Floresta Atlântica do Sudeste-Sul e Arremon franciscanus (Raposo, 1997) Tico-tico-do-são-francisco, restrito a região do alto e médio São Francisco. A. taciturnus é um modelo biológico interessante para o objetivo da pesquisa devido a sua distribuição e habitat de ocorrência. É uma espécie residente que ocorre apenas em ambiente florestal no nordeste brasileiro, com isso, sofre consequências negativas com a perda de habitat florestal. Além disso, espécies que são residentes e dependentes de ambientes florestais são mais vulneráveis a diversificações causadas por eventos vicariantes e, consequentemente isolamento geográfico (Porzecanski e Cracraft 2005).

\section{Área de estudo}

As atividades de campo foram realizadas em florestas altitude localizadas nos estados de Pernambuco e Paraíba, que se concentram em parte do planalto da Borborema e em áreas serranas nas depressões sertanejas Meridional e Setentrional, ecorregiões da caatinga (Veloso et al., 2002).

As atividades de inventário e coleta foram realizadas em quatro áreas de floresta de altitude, duas situadas ao leste da Borborema, são elas: Pico do Jabre, Maturéia, Paraíba; São João do Tigre, Paraíba.

\section{Amostragem de campo}

As amostragens sonoras acompanharam as atividades de campo do projeto "Relações biogeográficas entre a avifauna de florestas de altitude no nordeste do Brasil", projeto este financiado pelo Edital MCT/CNPq No 014/2010 - Universal, de acordo com o processo N$^{\circ} 478907 / 2010-9$ e executado pelo prof. Dr. Erich Mariano, em parceria com o prof. Dr. Helder Araújo da UFPB, campus de Areia. As atividades do presente trabalho estão vinculadas a licença de pesquisa número 24541 1 do SISBIO.

Embora a região da caatinga apresente uma variação sazonal marcante, as amostragens de campo foram realizadas no período chuvoso nas localidades inseridas no seu domínio. Tal estação de amostragem refere-se ao período reprodutivo da avifauna na região, quando os contatos sonoros com as espécies são mais frequentes (Araújo, 2012).

\section{Análises bioacústicas}

As gravações foram realizadas através do uso do gravador digital Marantz modelo PMD 660, acoplado a um microfone unidirecional de marca Senheiser. Para efeito de analise foram utilizados os cantos que apresentaram melhor qualidade de gravação, ou seja, aqueles que não tiveram muito ruído e não saturaram, de modo a permitir uma análise adequada.

Os cantos coletados foram transferidos para o computador no formato de $\mathrm{mp} 3$, posteriormente convertidos para o formato WAV e filtrados utilizando o software Cool Edit. O filtro foi aplicado para remoção de frequências inferiores a 7 
$\mathrm{kHz}$, não prejudicando a análise do canto, pois a frequência mínima do canto do A. taciturnus é maior que $7 \mathrm{kHz}$. Os cantos foram analisados por meio de espectrogramas (frequência $\mathrm{x}$ tempo) e oscilogramas (amplitude $\mathrm{x}$ tempo) através do software Sound Ruler.

Para análise sonora foram medidos parâmetros do canto, como: duração (s), número de notas, frequência dominante $(\mathrm{Hz})$, frequência fundamental $(\mathrm{Hz})$, amplitude $(\mathrm{Hz})$, e os seguintes parâmetros para notas: Duração (s), frequência inicial $(\mathrm{Hz})$, frequência final $(\mathrm{Hz})$, e amplitude $(\mathrm{Hz})$.

Inicialmente foi utilizado o teste de Lilliefors, uma modificação do teste de Kolgomorof-Smirnoff, para testar a normalidade dos dados. Foi utilizado o teste t no caso de dados normais, que é um teste paramétrico, para verificar se a diferença entre os cantos das formações florestais a oeste e leste do planalto da Borborema foi significativa ou não.

No caso em que os dados não foram normais, utilizamos o teste $\mathrm{U}$, que é um teste não paramétrico, para comparar as variáveis entre as florestas de altitude estudadas.

\section{Análises da biometria}

Foram realizados padrões de discriminação morfométricas entre as populações por meio da análise das variáveis canônicas ou análise discriminante múltipla (Marcus, 1990) e Análise de Correspondência. O grau de contribuição de cada caráter será definido pela correlação de Pearson $(P<0,05)$, obtidos a partir dos valores individuais gerados pelo banco de dados com as medidas morfométricas. A análise discriminante mostra-se bastante apropriada para estudos taxonômicos uma vez que contribuem para a identificação das espécies (Cavalcanti e Lopes, 1993).

Os dados morfométricos destas populações foram obtidos através das capturas por redes de neblina e por coleta com espingarda de ar comprimido. Os indivíduos coletados encontram-se na Coleção Ornitológica da Universidade Federal da Paraíba (UFPB), campus Areia. Para cada indivíduo coletado, foram medidas as seguintes variáveis: Asa, tarso, comprimento da cabeça, cúlmen, narina-ponta, diâmetro da base do bico, altura do bico, cauda, e comprimento total. Para o cálculo da análise discriminante foi utilizado o software Statistica 7.0 e para a Análise de Correspondência foi utilizado o MVSP 3.21.

\section{Resultados e discussão}

\section{Análises bioacústicas}

De forma geral, a espécie apresenta um canto com frequência dominante de $8.947,69 \mathrm{~Hz}$, uma média de 5,07 notas e divide-se em duas partes, sendo a primeira constituída de notas curtas, e a segunda com notas longas (Figuras 1 e 2). Quando comparamos o canto entre Florestas de altitude a leste e oeste do Planalto da Borborema, verificamos diferenças entre alguns parâmetros sonoros.

Na região leste da Borborema, o canto de A. taciturnus variou de 4 a 5 notas, tendo uma duração média de $1,77 \mathrm{~s}$, frequência dominante de $8.850,79 \mathrm{~Hz}$ e amplitude $3.599,69$ Hz. Na região oeste da Borborema, o canto apresentou uma maior duração média com 2,19 s, e também uma maior variação no número de notas, variando de 3 até 7 notas, sendo a sua maioria com 6 notas, e apenas um canto apresentou três notas. A frequência dominante nesta área foi de $8.803,98 \mathrm{~Hz}$, e a amplitude de $5.432,79 \mathrm{~Hz}$.
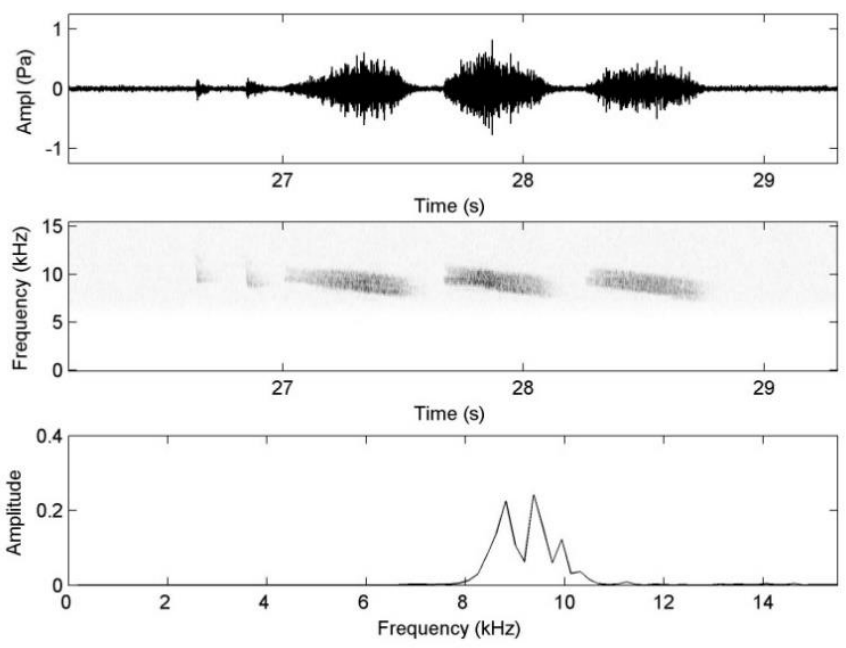

Figura 1. Sonograma referente a vocalização de $A$. taciturnus. $\mathrm{A}=$ Oscilograma; $\mathrm{B}=$ Espectrograma; $\mathrm{C}=$ Espectro da Amplitude.

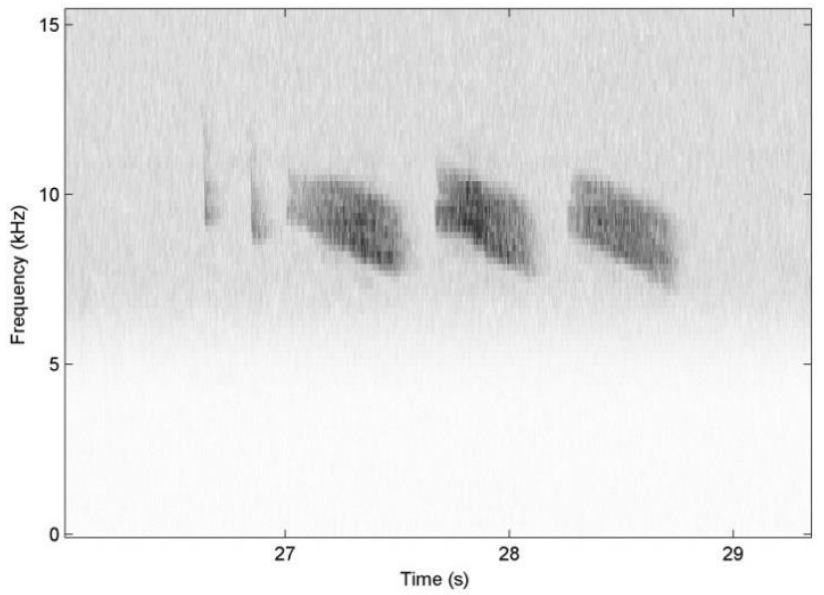

Figura 2. Espectrograma ilustrando as duas partes do canto de $A$. taciturnus. $\mathrm{kHz}=$ kilo Hertz, s = segundos.

Na região leste da Borborema os cantos apresentaram apenas duas notas na primeira parte do canto, e na segunda parte variaram de duas a três notas. Já na região Oeste da Borborema houve uma maior variação, podendo ser notada a ausência de notas na primeira parte de alguns cantos, sendo assim, as notas da primeira parte do canto variaram de 0 a 4 notas, e as notas da segunda parte variaram de 2 a 5 notas (Tabela 1). A presença e ausência de notas curtas introdutórias e variações no número de notas em populações de A. taciturnus também foram observadas em outro estudo (Buainain, 2017). No entanto, o mesmo autor não considerou como uma variação geográfica consistente. 
Tabela 1. Média e desvio padrão dos tipos de notas que constituem o canto das populações de Arremon taciturnus localizadas a Leste e Oeste do planalto da Borborema.

\begin{tabular}{|c|c|c|c|c|c|c|c|c|c|c|}
\hline \multicolumn{11}{|c|}{ Primeira parte } \\
\hline \multirow{2}{*}{$\mathrm{N}$} & \multirow{2}{*}{ notas } & \multirow{2}{*}{ local } & \multicolumn{2}{|c|}{ duração (s) } & \multicolumn{2}{|c|}{ f. inicial $(\mathrm{Hz})$} & \multicolumn{2}{|c|}{ f. final $(\mathrm{Hz})$} & \multicolumn{2}{|c|}{ amplitude (Hz) } \\
\hline & & & $\mathrm{X}$ & $\mathrm{S}$ & $\mathrm{X}$ & $\mathrm{S}$ & $\mathrm{X}$ & $\mathrm{S}$ & $\mathrm{x}$ & $\mathrm{S}$ \\
\hline 24 & 1 & Leste & 0,11 & 0,02 & 10933,31 & 527,93 & 8982,67 & 222,9171 & 1950,68 & 459,939 \\
\hline 20 & 1 & Oeste & 0,07 & 0,02 & 12475,77 & 959,49 & 9100,83 & 199,1 & 3374,93 & 1018,98 \\
\hline 20 & 2 & Oeste & 0,07 & 0,01 & 12438,8 & 744,42 & 8610,53 & 1397,52 & 3530,74 & 727,22 \\
\hline 20 & 3 & Oeste & 0,07 & 0,01 & 11621,55 & 2792,62 & 8868,31 & 124,85 & 3404,69 & 822,09 \\
\hline 20 & 4 & Oeste & 0,08 & 0,01 & 12842,6 & 517,33 & 8620,6 & 184 & 4260,87 & 598,72 \\
\hline
\end{tabular}

Segunda parte

\begin{tabular}{ccccccccccc}
\hline \multirow{2}{*}{$\mathrm{N}$} & \multirow{2}{*}{ notas } & \multirow{2}{*}{ local } & \multicolumn{2}{c}{ duração $(\mathrm{s})$} & \multicolumn{2}{c}{ f. inicial $(\mathrm{Hz})$} & \multicolumn{2}{c}{ f. final (Hz) } & \multicolumn{2}{c}{ amplitude (Hz) } \\
& & & $\mathrm{x}$ & $\mathrm{S}$ & $\mathrm{x}$ & $\mathrm{S}$ & $\mathrm{x}$ & $\mathrm{S}$ & $\mathrm{x}$ & $\mathrm{S}$ \\
\hline 27 & 1 & Leste & 0,46 & 0,060 & 10695,49 & 292,5615 & 8251,17 & 170,9405 & 2444,32 & 320,865 \\
33 & 1 & Oeste & 0,46 & 0,090 & 11126,15 & 471,72 & 7317,4 & 390,77 & 3808,77 & 794,36 \\
27 & 2 & Leste & 0,41 & 0,082 & 10632,17 & 270,223 & 8147,20 & 320,8651 & 2484,96 & 320,865 \\
33 & 2 & Oeste & 0,42 & 0,040 & 11051,06 & 505,92 & 7201,59 & 399,38 & 3858,53 & 813,9 \\
15 & 3 & Leste & 0,37 & 0,068 & 10427,46 & 259,451 & 7972,14 & 268,4982 & 2455,26 & 243,014 \\
30 & 3 & Oeste & 0,37 & 0,040 & 10981,82 & 656,16 & 6753,31 & 380,61 & 4229,17 & 881,93 \\
15 & 4 & Oeste & 0,36 & 0,040 & 11035,65 & 632,32 & 6702,38 & 213,61 & 4293,29 & 638,2 \\
6 & 5 & Oeste & 0,35 & 0,030 & 11121,15 & 271,79 & 6750,32 & 138,3 & 4372,34 & 381,01 \\
\hline
\end{tabular}

$\mathrm{N}=$ Número de cantos analisados, $\mathrm{x}=$ média, $\mathrm{S}=$ desvio padrão, f. inicial = frequência inicial, f. final = frequência final, Hz = Hertz.

O canto de $A$. taciturnus mostrou um maior número de notas e mais longa duração na região oeste da Borborema, quando comparada com a região leste. De acordo o teste $U$, foi verificada uma diferença significativa na amplitude e no número de notas do canto, no entanto, não houve nenhuma diferença entre as regiões na frequência dominante e na frequência fundamental. Para os dados da duração do canto, os quais apresentaram distribuição normal, o teste $\mathrm{t}$ evidenciou uma diferença significativa entre as mesmas regiões (Tabela 2).

Quando analisamos os diferentes tipos de notas separadamente através do teste t e do teste $\mathrm{U}$, apenas na duração das notas longas não obtivemos diferença significativa (Tabela 3).

Tabela 2. Comparação de parâmetros acústicos do canto de Arremon taciturnus entre as duas regiões estudadas.

\begin{tabular}{cccccc}
\hline Teste $\mathrm{P}$ e valor de $\mathrm{N}$ & duração & Freq. dominante & Freq. fundamental & amplitude & $\mathrm{n}^{\mathrm{o}}$ notas \\
\hline Teste & $\mathrm{t}=-5,766$ & $\mathrm{U}=287,0$ & $\mathrm{U}=287,0$ & $\mathrm{U}=6,0$ & $\mathrm{U}=185,5$ \\
$\mathrm{P}$ & 0,00 & 0,018516 & 0,018516 & 0,00 & 0,000 \\
\hline $\mathrm{N}$ & \multirow{2}{*}{$27 / 33$} & $27 / 33$ & $27 / 33$ & $26 / 33$ & $27 / 33$ \\
\hline Leste/Oeste) & & & & & \\
\hline
\end{tabular}

De acordo com as variações do ambiente e as adaptações geradas para eles, as aves tendem a modular suas vocalizações para maximizar a transmissão dos sinais acústicos (Blumstein e Turner, 2005; Tubaro e Lijtmaer, 2006; Seddon, 2005, Nicholls e Goldizen, 2006). Espécies que habitam o solo em florestas, geralmente, apresentam vocalizações com baixa frequência, o que possibilita ao sinal transpor barreiras impostas ao som, tais como a degradação do sinal devido à reverberação (Nicholls e Goldizen, 2006). Em áreas abertas às vocalizações costumam ser trinadas e rápidas, permitindo ao vento levar a informação por longas distâncias (Krebs e Davies, 1996; Nicholls e 24
Goldizen, 2006). Segundo a Hipótese de Adaptação Acústica as espécies tendem a maximizar a transmissão sonora a um gasto menor de energia (Blumstein e Turner, 2005; Tubaro e Lijtmaer, 2006; Seddon, 2005; Nicholls e Goldizen, 2006). Isso implica na modificação dos parâmetros acústicos, a fim de garantir a comunicação sonora a um menor gasto energético.

Em São João do Tigre, as formações florestais amostradas se apresentavam como vários pequenos fragmentos de não mais que $100 \mathrm{~m}^{2}$ circundadas por áreas de pasto e plantação. Por outro lado, a região dos Brejos de Bonito e da Mata do Pau Ferro apresentam fragmentos florestais maiores e mais conectados. 
Tabela 3. Resultado das comparações entre os parâmetros acústicos das notas do canto das populações de A. taciturnus localizadas a leste e oeste do Planalto da Borborema através dos testes $t$ e U.

\begin{tabular}{ccccc}
\hline $\begin{array}{c}\text { Parâmetro } \\
\text { Acústico }\end{array}$ & $\begin{array}{c}\text { Parte do } \\
\text { canto }\end{array}$ & $\begin{array}{c}\text { Tipo de } \\
\text { nota }\end{array}$ & Teste & $\mathrm{P}$ \\
\hline Nt1C- Dur. & $1^{\circ}$ parte & Curta & $\mathrm{t}=5,022$ & 0,000 \\
Nt1C- Ampl. & $1^{\circ}$ parte & Curta & $\mathrm{t}=-6,147$ & 0,000 \\
Nt2C- Dur. & $1^{\circ}$ parte & Curta & $\mathrm{U}=64,500$ & 0,000 \\
Nt2C- Ampl. & $1^{\circ}$ parte & Curta & $\mathrm{t}=-8,469$ & 0,000 \\
Nt1L- Dur. & $2^{\circ}$ parte & Longa & $\mathrm{t}=-0,149$ & 0,882 \\
Nt1L- Ampl. & $2^{\circ}$ parte & Longa & $\mathrm{U}=45,000$ & 0,000 \\
Nt2L-Dur. & $2^{\circ}$ parte & Longa & $\mathrm{U}=$ & 0,096 \\
& & & 333,500 & \\
Nt2L- Ampl. & $2^{\circ}$ parte & Longa & $\mathrm{U}=5,000$ & 0,000 \\
Nt3L- Dur. & $2^{\circ}$ parte & Longa & $\mathrm{t}=-0,179$ & 0,859 \\
Nt3L- Ampl. & $2^{\circ}$ parte & Longa & $\mathrm{t}=-7,607$ & 0,000 \\
\hline
\end{tabular}

$\mathrm{U}$ - valores do teste $\mathrm{U}, \mathrm{t}$ - valores do teste $\mathrm{t}$. $\mathrm{Nt} 1 \mathrm{C}$ - primeira nota curta, $\mathrm{Nt} 2 \mathrm{C}$ - segunda nota curta, Nt3C - terceira nota curta, $\mathrm{Nt} 1 \mathrm{~L}$ - primeira nota longa, $\mathrm{Nt} 2 \mathrm{~L}$ - segunda nota longa, $\mathrm{Nt} 2 \mathrm{~L}$ - terceira nota longa, Dur - duração, Ampl = amplitude.

Um exemplo de estudo realizado que corroborou com esta hipótese foi um trabalho com a espécie de ave Herpsilochmus longirostris (Pelzeln, 1868) Chorozinho-de-bico-comprido, onde foram comparadas variações em parâmetros acústicos de populações em área natural e em parque (Mendonça, 2009). No entanto, seria necessário um estudo mais detalhado e comparativo com áreas impactadas e não impactadas para melhor entendimento dos efeitos do ambiente causado nos parâmetros acústicos das populações de A. taciturnus.

Além da adaptação acústica, um outro fator que resulta em pequenas variações nos cantos de determinadas espécies é o isolamento geográfico, provocando assim a formação de dialetos vocais (Silva, 1995). Para aves como A. taciturnus que possui uma capacidade de aprendizado vocal e ainda é dependente de ambientes florestais (Stotz, 1996), é possível que pequenas variações encontradas entre as populações a leste e oeste do planalto seja consequência do isolamento e falta de contato sonoro entre estas populações. Alguns trabalhos discutem que mudanças em características fenotípicas (morfológicas e vocais) podem surgir numa população em curtos períodos de tempo, ou até em poucas gerações através de pressões seletivas (Grant e Grant, 2009), a exemplo do surgimento de barreiras geográficas.

As espécies de aves que possuem seus cantos definidos de forma genética, ou inato, apresentam uma estrutura física no canto bastante estereotipada. Isso permite com que a estrutura seja mantida de geração para geração, permanecendo os parâmetros específicos de reconhecimento mesmo na ausência de um modelo (Vielliard, 1986, 1987). Por ser um caráter conservador, o canto inato evolui devagar de acordo com a influência da deriva genética e pressões do meio. Em prática, a evolução filogenética nestas espécies seria bem aparente, mas isto não impede que ocorra uma evolução adaptativa. No entanto, a variação individual e populacional nestas espécies é bastante limitada, mesmo que populações se encontrem isoladas por milhares de gerações (Vielliard, 1986, 1987; Sales, 2016). Por outro lado, as espécies que apresentam capacidade de aprendizado como o A. taciturnus, devem apresentar um maior grau de variação geográfica, pelo fato de haver rápida evolução cultural entre populações. Esse fato foi demonstrado por exemplo nos Oscines (Saranathan et al., 2007) e nos psitacídeos (Wright, 1996). Apesar destas espécies apresentarem uma parte das características do canto determinada geneticamente, a aprendizagem consiste em moldar este padrão a um modelo, muitas vezes adquirido pela imitação de seus tutores nos primeiros momentos de vida seguido de uma fase de treino e ajustamento no início da primeira reprodução (Vielliard, 1986; Silva, 2011) e com isso, consequentemente surgem variações populacionais e até individuais (Silva, 1995; Silva, 2011).

\section{Análises biométricas}

Os indivíduos de A. taciturnus coletados nas regiões estudadas apresentaram, de um modo geral, as seguintes medidas: Asa (média $=70,29 ; \quad \mathrm{S}=4,18$ ), tarso (média=24,67; $\mathrm{S}=1,33$ ), comprimento da cabeça (média $=32,17 ; \mathrm{S}=1,33$ ), cúlmen (média=27,10; $\mathrm{S}=40,07$ ), narina-ponta (média=10,71; $\mathrm{S}=1,38$ ), diâmetro da base do bico (média $=8,11 ; \mathrm{S}=1,05$ ), altura do bico (média $=7,14 ; \mathrm{S}=0,90$ ), e cauda (média $=61,42 ; \mathrm{S}=8,46$ ).

Tabela 4. Resultado da Análise discriminante dos dados biométricos das populações de A. taciturnus nas regiões leste e oeste do planalto da Borborema.

\begin{tabular}{lcccccc}
\hline & $\begin{array}{c}\text { Wilks' } \\
\text { Lambda }\end{array}$ & $\begin{array}{c}\text { Partial } \\
\text { Lambda }\end{array}$ & $\begin{array}{c}\text { F- } \\
\text { remove } \\
(1,23)\end{array}$ & p-level & Toler. & $\begin{array}{c}\text { 1-Toler. } \\
\text { (R-Sqr.) }\end{array}$ \\
\hline Asa & 0,693 & 0,998 & 0,046 & 0,833 & 0,640 & 0,360 \\
Tarso & 0,771 & 0,897 & 2,635 & 0,118 & 0,851 & 0,149 \\
Culmen & 0,694 & 0,996 & 0,082 & 0,777 & 0,377 & 0,623 \\
Narina-ponta & 0,708 & 0,976 & 0,555 & 0,464 & 0,350 & 0,650 \\
D. base bico & 0,706 & 0,980 & 0,464 & 0,502 & 0,632 & 0,368 \\
A. bico & 0,742 & 0,933 & 1,664 & 0,210 & 0,763 & 0,237 \\
Cauda & 0,694 & 0,996 & 0,087 & 0,771 & 0,668 & 0,332 \\
\hline
\end{tabular}




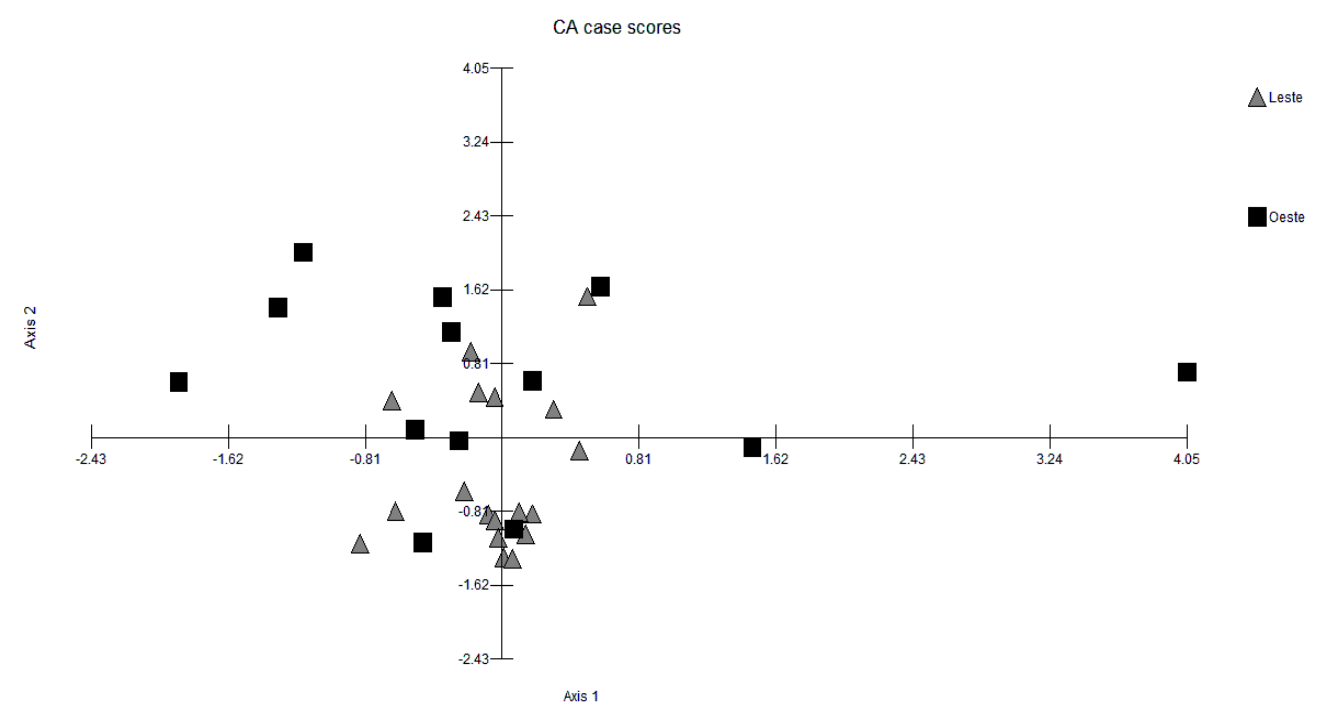

Figura 3. Resultado da análise da correspondência entre as populações de A. taciturnus localizadas a leste e oeste do Planalto da Borborema.

Não foi observado polimorfismo dentro das populações estudadas de A. taciturnus, entretanto, as análises morfométricas foram realizadas afim de se verificar possíveis sinais de modificações morfológicas entre as populações com sete das dez variáveis morfométricas aferidas. A redução das variáveis para estas análises se deu devido à ausência da informação em alguns exemplares da coleção da UFPB.

Na comparação entre os espécimes, apenas um eixo canônico estatisticamente significativo (critério de Wilks, $\mathrm{P}<0,05$ ) (tabela 4), e a análise de correspondência não separa os grupos populacionais (Figura 3 ).

\section{Conclusões}

Apesar do ambiente poder moldar parâmetros do canto de muitas espécies, devemos considerar que o A. taciturnus é uma espécie que possui uma capacidade de aprendizado vocal. Sendo assim, podemos concluir que algumas diferenças significativas nos parâmetros do canto são variações interespecíficas que podem ter surgido como consequência do isolamento geográfico (surgimento de dialetos vocais), já que a espécie ocorre em condições ambientais determinantes para sua presença e as populações estudadas estão separadas por uma barreira geográfica para estas populações, que também é suportada por outros trabalhos. Além disso, devemos considerar que mudanças em características fenotípicas (morfológicas e vocais) podem surgir numa população em curtos períodos de tempo, ou até em poucas gerações através de pressões seletivas a exemplo do surgimento de barreiras geográficas.

\section{Agradecimentos}

Ao CNPq pelo financiamento das atividades de campo. A UFCG, e colegas do Laboratório de Ornitologia e Biologia da Conservação da UFCG pela contribuição para a realização do presente trabalho.

\section{Referências}

Andrade-Lima, D. 1982. Present-day forest refuges In Northeastern Brazil. In Prance, G. T. (Ed.) Biological diversification in the tropics. Columbia University Press, New York, p.245-251. 714p.

Araujo H.F.P.; Vieira-Filho, A. H.; Cavalcanti, T. A.; Barbosa, M. R. V. 2012 As aves e os ambientes em que elas ocorrem em uma reserva particular no cariri paraibano, nordeste do Brasil. Revista Brasileira de Ornitologia, 20(3), 365-377.

Blumstein, D. T.; Turner, A.C. 2005. Can the acoustic adaptation hypothesis predict the structure of Australian bird song? Acta ethologica, 8:35-34.

Buainain, N.; Assis, C. P. A.; Raposo, M. 2017. Geographic variation and taxonomy of the Arremon taciturnus (Hermann, 1783) species complex (Aves: Passerellidae). Journal of Ornithology, 158(3): 631-650.

Cavalcanti, M. J.; Lopes, P. R. D. 1993. Análise morfométrica multivaridada de cinco espécies de serranidae (teleostei, perciformes). Acta biológica leopoldensia. 15: 53-64.

Goldizen, A. W.; Nicholls, J. A. 2006. Habitat type and density influence vocal signal design in satin bowerbird. Journal of animal, 75:549-558.

Grant, P. R. e GRANT, B. R. 2009. The secondary contact phase of allopatric speciation in Darwin's finches. Proceedings of the National Academy of Sciences of the United States of America, 106(48): 20141-8.

Kroodsma, D. E.; Baylis, J. R. 1982. Appendix: a world survey of evidence for vocal leraning in birds. In: Kroodsma, D. E.; Miler, E.H.(eds.) Acoustic communication in birds. New York, NY: Academic press. 2:311-337.

Lijtmaer, D. A.; Tubaro, P. L. 2006. Environmetal corelates of song structure in forest grosbeaks and saltators. The condor, 108: 120-129.

Marcus, L. F. Traditional morphometrics. 1990. In: Rohlf, F. J.; Bookstein, F. L. (eds.) Proceedings of the michigan morphometrics workshop. Michigan: The university of Michigan museum of zoology, special publication. 2:77122.

Mariano E. F. 2014. Relações biogeográficas Entre a avifauna de florestas de altitude no nordeste do Brasil. Tese de doutorado. Universidade Federal da Paraíba - UFPB. João Pessoa, Paraíba. 230p.

Marler, P. 2004. Science and birdsong: the good old days. In: Marler, P.; Slebberkoorn, H. (eds.) Nature's music the science of birdsong. San diego: Elsevier academic press. p.1-37.

Mendonça, E. T. 2009. A utilização de Herpsilochmus longirostirs Pelzeln, 1868 (passeriformes: Thamnophilidae) como modelo de adaptação acústica. Dissertação de mestrado. Universidade Federal de Uberlândia.

Mundinger, P. C. Microgeographic and macrogeographic variation. 1982. In: Kroodsma, D. E.; Miler, E.H. (eds.) Acoustic communication in birds. New York, NY: Academic press. 2:147-208.

Porzencanski A.L. e J. Cracraft. 2005. Cladistic analysis of distributions and endemism (CADE): using raw distributions of birds to unravel the biogeography of the South American aridlands. Journal of Biogeography 32: $261-275$.

Ridgely, R. S.; Tudor G. 2009. Songbirds of south America: The Passerines. 
University of Texas. 750p.

Rodal, M. J. N.; Barbosa, M. R. V.; Thomas, W. W. 2008. Do the seasonal forests in northeastern Brazil represent a single floristic unit? Brazilian journal of biology. 68 (3): 467-75.

Sales, R. G. 2016. Variação vocal de espécies da família Thamnophilidae (aves) associadas a barreiras ribeirinhas na Amazônia. Dissertação de mestrado, Programa de Pós-graduação em Biologia Animal, da Universidade Federal de Pernambuco. 83p.

Saranathan, V.; Hamilton D.; Powell, G. V. N.; Kroodsma D. E.; Prum R. O. 2007. Blackwell Publishing Ltd Genetic evidence supports song learning in the three-wattled bellbird Procnias tricarunculata (Cotingidae). Molecular Ecology. 16:3689-3702.

Seddon, N. 2005. Ecological adaptation and species recognition drives vocal evolution in neotropical suboscine bird. Evolution. 59:200-215.

Shieh, B. S.; Liang, S. H. 2007. Geographic variations and temporal changes in songs of the rufous-capped babbler (Stachyris ruficeps praecognita). Ornis fennica. 84:163-172.

Silva, M. L. 1995. Esteriotipia e versatilidade nos cantos das aves: os padrões de cantos em sabiás e outras aves. Anais de etologia, 13:133-147.

Silva, M. L.; Vielliard, J. M. E. A. 2011. aprendizagem vocal em aves: evidências comportamentais e neurobiológicas. In: Henriques A.; Assis, G,; Brito, R.; Martin, W. L. (Org). Estudos do comportamento II. Belém: Editora da UFPA.

Stotz, D. F.; Fitzpatrick, J.W.; Parker, T.A.; Moskovits, D.K. 1996. Neotropical Birds: ecology and conservation. Chicago and London: The University of Chicago Press. 478p.

Tubaro, P. L. 1999. Bioacústica aplicada a la sistemática, conservacion y manejo de poblaciones naturales de aves. Etologia, Buenos Aires, 7:19-32.

Veloso, A. L., Sampaio, E. V. S. B. e Pareyn, F. G. C. 2002. Ecorregiões propostas para o Bioma Caatinga. Associação Plantas do Nordeste; Instituto de Conservação Ambiental The Nature Conservancy do Brasil. 80p.

Vielliard, J.M.E. 1987. Uso da Bioacústica na observação de aves. In: II Encontro de Anilhadores de aves. Rio de Janeiro: Editora Universidade Federal do Rio de Janeiro. p. 98-121.

Vielliard, J.M.E. 1997. Uso de caracteres Bioacústicos para avaliações filogenéticas em aves. Anais de Etologia, 15: 93- 107.

Wright, T. F. 1996. Regional Dialects in the Contact Call of a Parrot. Proc. R. Soc. London. Ser. B Biol. Sci. 263: 867-872. 